\title{
The effect of depression on fracture healing and osteoblast differentiation in rats
}

This article was published in the following Dove Press journal:

Neuropsychiatric Disease and Treatment

\author{
Chunzi Nie' \\ Zhan Wang ${ }^{2}$ \\ Xufeng Liu' \\ 'Department of Military Medical \\ Psychology, Fourth Military \\ Medical University, Xi'an 7I0032. \\ People's Republic of China; \\ ${ }^{2}$ Department of Orthopaedics, Gansu \\ Provincial Hospital, Lanzhou, Gansu \\ 730050, People's Republic of China
}

Background: Depressive disorder has been proven to be associated with disturbed bone metabolism. However, the effect of depression on fracture healing still lacks evidence.

Materials and methods: A rat depressive model was first established by exposing the animals to chronic unpredictable stress, which was assessed using the sucrose preference test, forced swimming test, and open field test. Subsequently, the bone repairing ability was detected by micro-computed tomography and histological analysis of the femoral condyle defect rats with or without depression. To further investigate the potential mechanisms of depression on fracture healing, the osteogenic differentiation and autophagic level were compared between the bone marrow mesenchymal stem cells (BMSCs) derived from depressive and normal rats.

Results: Our results showed that rats with depressive disorder significantly slowed the healing process at 4 and 8 weeks postinjury. Furthermore, the osteogenic potential and autophagy remarkably decreased in BMSCs from the depressive rats, suggesting an inherent relationship between autophagy and osteogenic differentiation. Finally, rapamycin, an autophagic agonist, significantly improved osteogenic differentiation of depressive BMSCs through autophagy activation.

Conclusion: The present study indicated that depression had a negative effect on fracture healing with low osteoblast differentiation of BMSCs. Also, autophagy activation in BMSCs offers a novel therapeutic target for depressive patients with poor fracture healing.

Keywords: depression, fracture healing, osteoblast differentiation, autophagy, bone marrow mesenchymal stem cells

\section{Introduction}

Mental illness is now considered as a serious public health issue and is widespread throughout the world. More specifically, depressive disorders are the third leading cause for years lived with disability - a measure of nonfatal burden. ${ }^{1}$ A strong association between psychiatric and somatic disorders has been generally accepted. In recent years, accumulating evidence suggests that depression negatively influences bone metabolism. ${ }^{2}$ In a recent population-based cohort study, results have indicated that mental disorders and medication use are associated with an increased risk for fracture. ${ }^{3}$ However, the influence of depression on fracture healing still remains unclear.

Osteoblastic differentiation and osteoid deposition are the most important elements for fracture repair. In contrast, decreased bone formation is the key feature for poor bone healing. It is well known that osteoblasts with osteogenetic potential are mainly originated from bone marrow mesenchymal stem cells (BMSCs). ${ }^{4}$ Wan et $\mathrm{al}^{5}$ reported that defective autophagy of BMSCs led to the low osteogenic potential in patients with osteoporosis. Thus, we hypothesized that the changes of osteoblast differentiation and autophagy in BMSCs may be the underlying mechanism for the effect of depression
Correspondence: Xufeng Liu Department of Military Medical Psychology, Fourth Military Medical University, Xi'an 7I0032,

People's Republic of China Email Ixf_psychology@I63.com 
on fracture healing. Thus, this study set out to compare the bone healing ability of femoral condyle defect in rats with or without depression and further also investigated the autophagy changes of the BMSCs derived from depressive rats and whether the autophagy was responsible for maintaining its osteogenic capacity.

\section{Materials and methods Establishment of a rat depression model} In the present study, adult male Sprague Dawley rats (4 weeks old, 200-220 g weight) were purchased from Experimental Animal Center of Fourth Military Medical University. The study protocol was approved by the Institutional Animal Care Committee of Fourth Military Medical University and performed in compliance with the National Institutes of Health Guide for the Care and Use of Laboratory Animals. After the animals were adaptively fed and group-housed for a week, 40 Sprague Dawley rats were divided into 2 groups randomly (20/group). Rats in group A had normal feeding, while the others in group B suffered from chronic unpredictable stress (CUS). The normal feeding rats were housed in a single cage at a constant temperature $\left(23^{\circ} \mathrm{C}-25^{\circ} \mathrm{C}\right)$ and $55 \%-66 \%$ relative humidity with food and water available ad libitum on a 12-h light/dark cycle. Simultaneously, each rat in the group B was under CUS stimulation and these were also housed in a single cage. The CUS procedure consisted of the following: fasting for $24 \mathrm{~h}$, water deprivation for $24 \mathrm{~h}$, exposure to noise for $1 \mathrm{~h}(80 \mathrm{~dB})$, placement in wet litter for $24 \mathrm{~h}$, restricted activity for $1 \mathrm{~h}$, tilting of the cage to an angle of $45^{\circ}$ for $24 \mathrm{~h}$, placement in a $4^{\circ} \mathrm{C}$ cold water bath for $5 \mathrm{~min}$, placement in a $45^{\circ} \mathrm{C}$ hot water bath for $5 \mathrm{~min}$, tail clamp for 1 min, night lighting in the cage, and electric shock to the foot 5 min (current intensity $1.0-1.5 \mathrm{~mA}$ ), as reported previously. ${ }^{6}$ The stressors were applied in a random sequence, and this CUS procedure was administered to the stressed rats once per day. After 4 weeks, the changes in depressive behavior were assessed using the sucrose preference test (SPT), forced swimming test (FST), and open field test (OFT), following previously described methods. ${ }^{7}$

\section{Behavior tests}

The depression-like behaviors were evaluated by SPT, FST, and OFT. Rats were habituated to the testing room for more than half an hour before every test.

\section{SPT}

The SPT lasted for 5 days, and each rat was first single housed and habituated to a $2 \%$ sucrose solution for $48 \mathrm{~h}$.
On the third day, rats were allowed free access to 2 bottles containing $2 \%$ sucrose solution and tap water, respectively. To avoid bottle side preference, the 2 bottles were exchanged during an intermediate time point. The amount of liquid consumed from each of the bottles was measured after $72 \mathrm{~h}$, and the sucrose preference was calculated using the formula (the sucrose water intake)/(the tap water intake + the sucrose water intake) $\times 100 \%$.

\section{FST}

The FST was performed in a glass cylinder $(20 \mathrm{~cm}$ in diameter, $50 \mathrm{~cm}$ in height, and $30 \mathrm{~cm}$ water depth), with water temperature controlled at $24^{\circ} \mathrm{C} \pm 1^{\circ} \mathrm{C}$. The FST procedure included a 15 min pretest swim and a 5 min test swim after $24 \mathrm{~h}$. The immobility time was recorded with a stopwatch when the rat was floating with all limbs, motionless, during the 5 min formal test.

\section{OFT}

Each rat was placed in the center area of an open field apparatus which consisted of a clear Plexiglas box (100x $100 \times 40 \mathrm{~cm}^{3}$, with its floor divided into 25 equal-size squares). After $1 \mathrm{~min}$ adaptation, the rats were allowed to explore the area for $5 \mathrm{~min}$. The accumulative distance, the time spent in the central area, and the frequencies of rearing (defined as standing upright on the hind legs) were recorded using a digital camera.

\section{Establishment of femur defect model}

Once the successful establishment of the depression model was confirmed by behavior evaluation, 10 rats from each group was collected to establish the femur defect model according to procedures as previously described. ${ }^{8}$ Briefly, each rat was given a general anesthetic through an intravenous administration of chloral hydrate $(10 \%, 5.0-7.5 \mathrm{~mL} / \mathrm{kg})$. Then, a $1.5 \mathrm{~cm}$ incision was made to expose the genu after shaving and disinfection. A bone defect $1 \mathrm{~mm}$ in diameter and $2 \mathrm{~mm}$ in depth was drilled using a medical electrical drill in the central portion of the femoral condyle. Finally, the incision was sewn closed and antibiotics and analgesics were injected intramuscularly. The bone defect healing was assessed by micro-computed tomography (CT) analysis and histological observation at 4 and 8 weeks postsurgery.

\section{Micro-CT analysis}

New bone formation in the defect region of femoral condyles was monitored using an animal micro-CT scanner (SkyScan 1176, Bruker-micro-CT, Kontich, Belgium). 
Briefly, the treated rats were anesthetized and placed on the scanning table. The scanning conditions were kept identical for all the tests (spatial resolution $18 \mu \mathrm{m}, \mathrm{X}$-ray voltage $60 \mathrm{kV}$, exposure time $600 \mathrm{~ms}$, aluminum filter $1.0 \mathrm{~mm}$ ). Volumetric reconstructions and analyses were performed using built-in software NRecon 1.6 (Bruker-micro-CT) and CTAn 1.8 (Bruker-micro-CT), and the 3D reconstruction was built using CTvox software (Bruker-micro-CT). The bone volume/total volume ratio (BV/TV, \%), trabecular number (Tb.N), trabecular thickness (Tb.Th), and trabecular separation ( $\mathrm{Tb} . \mathrm{Sp}$ ) of the volume of interest were determined, which was a cylindrical area covering the femoral condyle bone defect.

\section{Histological observation}

After micro-CT scanning, 5 rats from each group were euthanized and bilateral femurs were removed for histological observation. Briefly, the femoral specimens were first fixed in $4 \%$ paraformaldehyde for $48 \mathrm{~h}$ and then soaked in 17\% EDTA at pH 7.3 until complete decalcification. Then, the decalcified specimens were embedded in paraffin and further cut into $5 \mu \mathrm{m}$ sections for hematoxylin and eosin (HE) staining. New bone formation was identified under light microscopy (Eclipse 80i, Nikon, Tokyo, Japan).

\section{Isolation and cultivation of BMSCs}

The remaining 10 rats from each group with or without depression were used to isolate BMSCs as previously described. ${ }^{9}$ Briefly, rats were euthanized and bilateral femurs were removed. The marrow was extruded by flushing with excessive amounts of $\alpha$-Dulbecco's Modified Eagle's Medium ( $\alpha$-DMEM, Gibco, Langley, OK, USA), and the bone marrow aspirated was subjected to density gradient centrifugation to collect the mononuclear cells. Then, these cells were seeded into a $25 \mathrm{~cm}^{2}$ plastic flask and cultured in $\alpha$-DMEM containing 10\% fetal bovine serum (FBS, Gibco) at $37^{\circ} \mathrm{C}$ in a humidified $5 \% \mathrm{CO}_{2}$ atmosphere. After 3 days culture, the nonadherent cells were removed by changing the culture medium, and the adherent cells were further cultured and the medium was refreshed every 2 days. The BMSCs were passaged upon reaching $80 \%-90 \%$ confluence, and cells from the third passage (P3) were used for experiments.

\section{Osteoinductive culture of BMSCs}

The P3 BMSCs were switched to the osteoblastic differentiation media, which was made up of the following: $0.1 \mu \mathrm{M}$ dexamethasone, $10 \mathrm{mM} \beta$-glycerolphosphate, and $0.25 \mathrm{mM}$ ascorbate (Sigma-Aldrich, St Louis, MO, USA) in $\alpha$-DMEM with $10 \%$ FBS for continuous culturing for 21 days culture. The medium was changed every 3 days. Then, the osteogenic potential was compared between BMSCs derived from rats with or without depression, which was detected by alkaline phosphatase (ALP) and Alizarin red $\mathrm{S}$ (ARS) staining as previously described. ${ }^{10}$

Furthermore, the osteogenesis-related markers like collagen type I (COL I), osteocalcin (OCN), osteopontin (OPN), and Runt-related transcription factor 2 (RUNX2) were measured with real-time quantitative polymerase chain reaction (qPCR). GAPDH was amplified as an internal control. All the rat primer sequences are listed in Table 1. Briefly, total RNA was extracted from BMSCs after osteogenic induction by using Trizol reagent (Invitrogen, Waltham, MA, USA). Then, $1 \mu \mathrm{g}$ of mRNA was reverse transcribed to cDNA, which was amplified by real-time PCR using the SYBR ${ }^{\circledR}$ Green Real-Time PCR Kit (Takara, Tokyo, Japan) according to the manufacturer's protocol. The relative amounts of target genes were calculated by $\Delta \Delta C_{\mathrm{t}}$ method.

\section{Cell autophagy analysis}

The basic autophagic level was first compared prior to osteogenic induction in P3 BMSCs. To visualize the autophagosome, transmission electron microscopy (TEM) was performed according to operator instructions. Briefly, cells were collected and fixed with $2.5 \%$ glutaraldehyde and then incubated with $1 \%$ osmium tetroxide for $2 \mathrm{~h}$ after washing with distilled water. The specimens were further counterstained with lead citrate and uranyl acetate, dehydrated in ethanol, and embedded in Epoxy-resin. Ultrathin sections $(50 \mathrm{~nm})$ were cut for final observation using a Hitachi-7500 TEM (Tokyo, Japan).

Table I Primer sequence of target gene for rats

\begin{tabular}{|c|c|c|}
\hline Gene & Forward primer $\left(5^{\prime}-3^{\prime}\right)$ & Reverse primer $\left(5^{\prime}-3^{\prime}\right)$ \\
\hline Coll & TCCGGCTCCTGCTCCTCTTA & GGCCAGTGTCTCCCTTG \\
\hline OCN & AGCCACCGAGACACCATGAGA & AGCCACCGAGACACCATGAGA \\
\hline OPN & CATACAAGGCCATCCCCGTT & ACGGCTGTCCCAATCAGAAG \\
\hline RUNX2 & ACTACCAGCCACCGAGACCA & ACTGCTTGCAGCCTTAAATGACTCT \\
\hline GAPDH & CAATGACCCCTTCATTGACC & TGGACTCCACGACGTACTCA \\
\hline
\end{tabular}


The autophagy-associated proteins were also determined by Western blot. Briefly, cells were lysed on ice using RIPA Lysis buffer (Beyotime, Jiangsu, People's Republic of China) to obtain the total cellular protein. Equal amounts of protein were separated by SDS-PAGE gels and transferred onto PVDF membranes, which were further locked with blocking buffer (Abcam, Cambridge, UK) for $1 \mathrm{~h}$ at $37^{\circ} \mathrm{C}$. Membranes were incubated with diluted primary antibody [1:1,000 LC3, P62, Abcam;1:5,000 $\beta$-actin, Bioss, Shanghai, People's Republic of China] at $4^{\circ} \mathrm{C}$ overnight. Then, the membranes were washed with TBST buffer and incubated with the respective secondary antibodies for $1 \mathrm{~h}$ at $37^{\circ} \mathrm{C}$. Finally, the bands were visualized using the enhanced ECL detection kit (Bio-Rad, Hercules, CA, USA).

To further investigate the influence of autophagy on osteogenic differentiation, the depressive BMSCs were treated with $100 \mathrm{nM}$ rapamycin (RAP) (Sigma) for $24 \mathrm{~h}$ prior to osteogenic induction in depressive BMSCs to induce autophagy.

\section{Statistical analysis}

All experiments were performed in triplicate, and data were expressed as mean \pm standard deviation (SD). SPSS version 19.0 statistical software program (IBM Corporation, Armonk, NY, USA) was used for statistical analysis. Differences between groups were analyzed by independent $t$-test or analysis of variance followed by Tukey's post hoc test. A $P$-value of $<0.05$ was considered as statistically significant.

\section{Results \\ CUS caused depression-like behavior in rats}

The schedule of this experimental design is shown in Figure 1A. The SPT is a typical depression-like behavior test for anhedonia in rats. At the end of the fifth week, results showed that the rats that had undergone CUS protocol had a significant decline in their sucrose preference than those in normal feeding group. Also, sucrose consumption

A

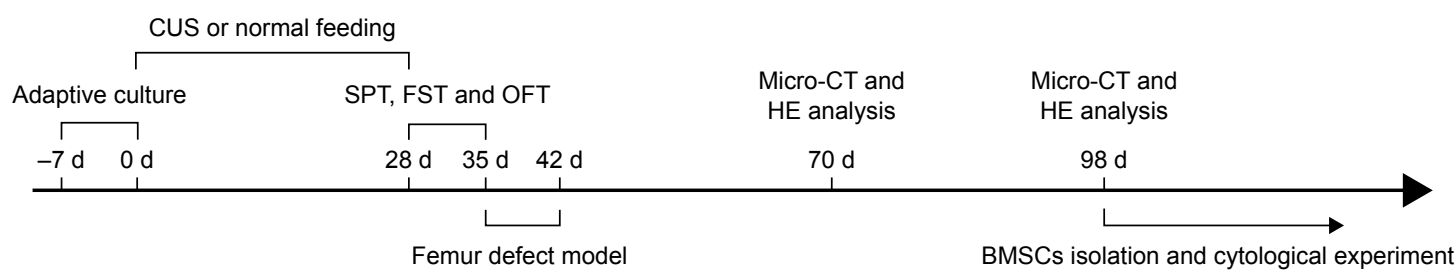

B

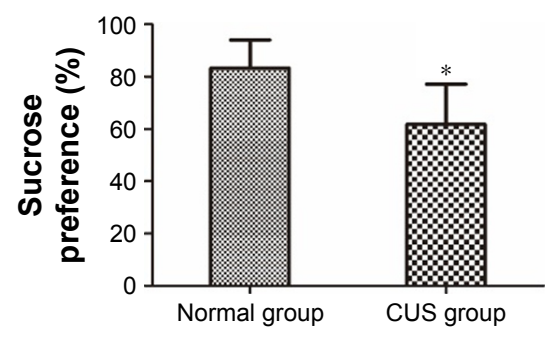

D

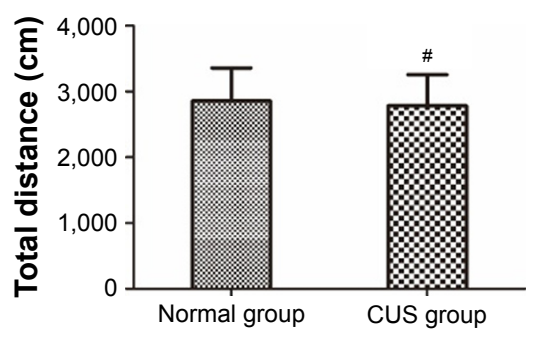

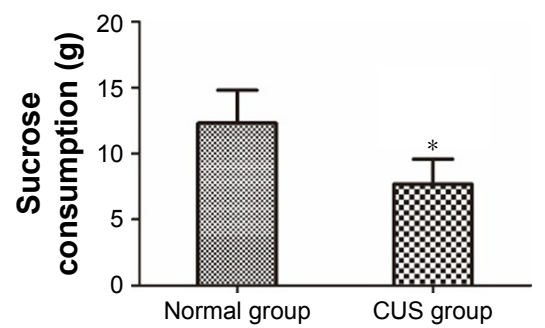

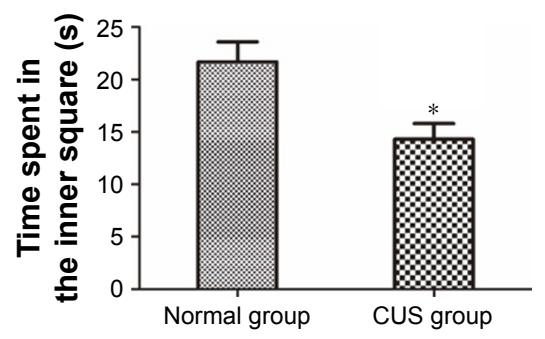

C
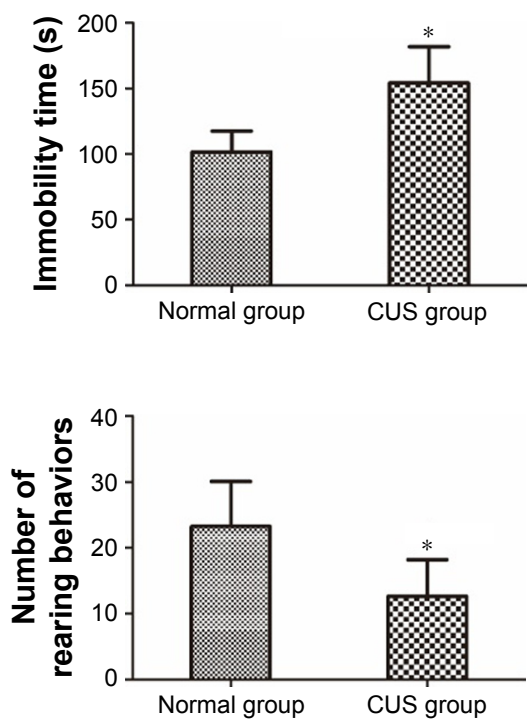

Figure I CUS caused depression-like behavior in rats.

Notes: (A) Experimental flowchart. (B) The sucrose preference and sucrose consumption were detected by SPT. (C) The immobility time in a beaker of water was detected by FST. (D) The total distance, time spent in center, and number of rearing behaviors were detected by OFT. The values are expressed as the mean \pm SD. $* P<0.05$ vs normal group. ${ }^{*}>0.05$ vs normal group.

Abbreviations: CUS, chronic unpredictable stress; SPT, sucrose preference test; OFT, open field test; FST, forced swimming test; CT, computed tomography; $\mathrm{HE}$, hematoxylin and eosin; BMSCs, bone marrow mesenchymal stem cells. 
decreased markedly in the depressive rats (Figure 1B). In the FST, immobility time in a beaker of water was significantly increased in the depressive rats compared with normal ones (Figure 1C). Furthermore, OFT analysis, usually conducted to measure spontaneous activity in rat, indicated the time spent in center and number of rearing instances were both significantly decreased in CUS-treated rats, but did not affect results of the total distance (Figure 1D). Taken together, these results proved that CUS successfully induced depression-like behaviors without influencing mobility.

\section{Depression attenuated bone healing in a rat femur defect model}

$3 \mathrm{D}$ reconstruction was used to evaluate the new bone formation within femur defect in rats. The results showed that there was visible bone formation 4 weeks postsurgery in normal feeding rats; however, little bone was formed in the center of the defect in depressive rats at the same time. Later, rats from group A showed complete bone repairing in the defect region at 8 weeks postsurgery, but an apparent defect still persisted in the rats from group B (Figure 2A). Quantification of various micro-CT parameters showed that the bone microarchitecture was significantly attenuated in depressive rats, of which BV/TV of new bone volume significantly decreased. The decrease in BV/TV of rats in group B was accompanied by lower Tb.N and Tb.Th, but higher Tb.Sp, as shown in Figure 2B.

HE staining was performed to further investigate the morphology of bone healing within the femur defects. At 4 weeks postsurgery, the bone defect region was occupied by fibrous tissue in group A. Simultaneously, apparent new bone formation from the center of the defect could be detected in group B. At 8 weeks postsurgery, there was obviously newly formed bone, which was increasing, within
A

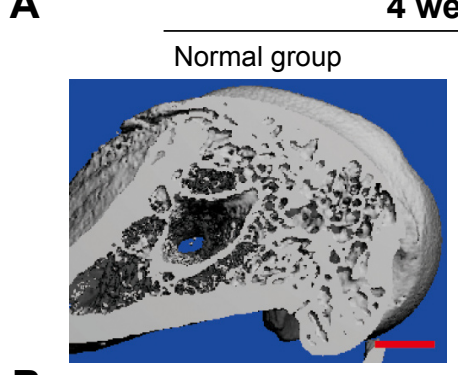

4 weeks

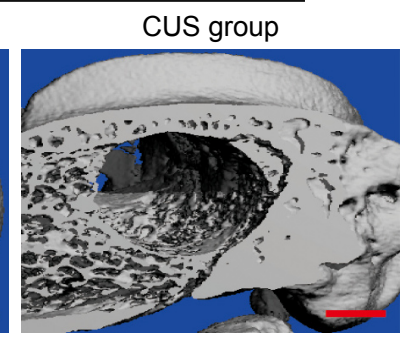

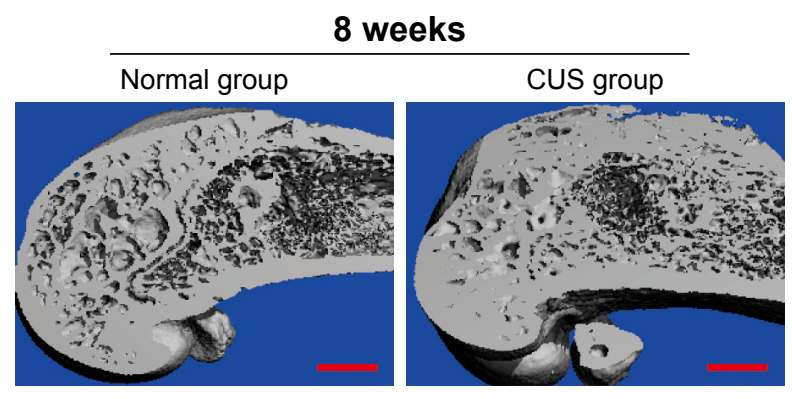

B
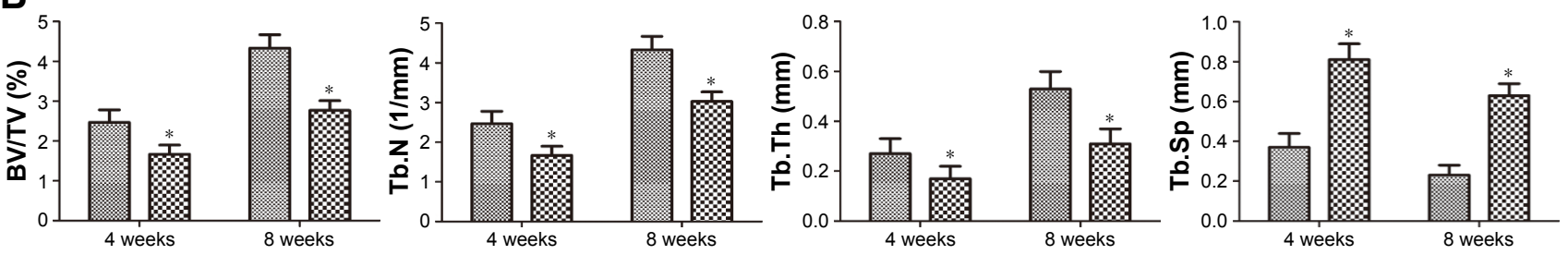

Normal group $\quad$ CUS group

C

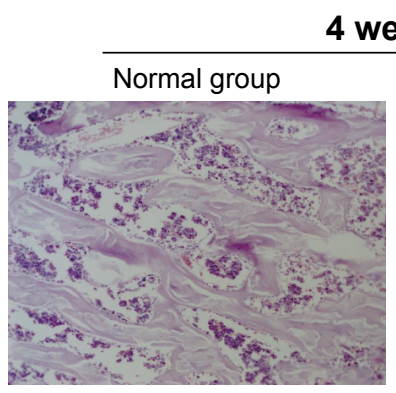

4 weeks

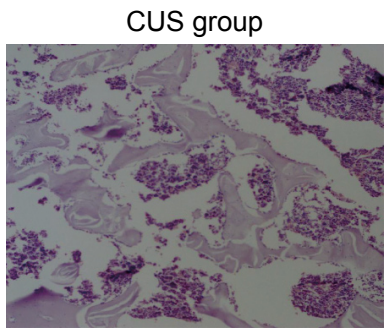

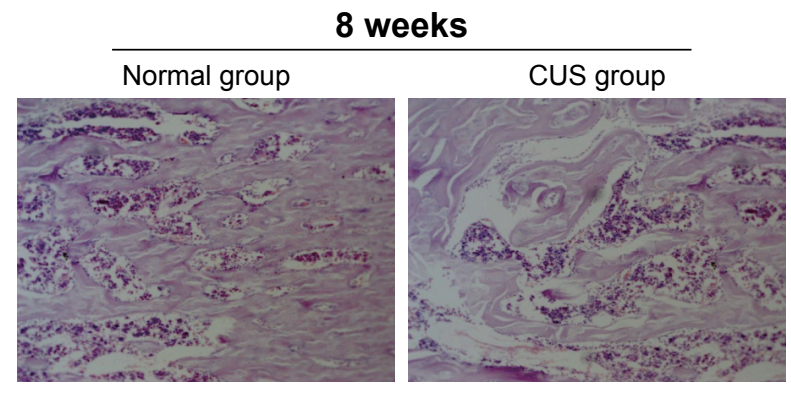

Figure 2 Depression attenuated bone healing in a rat femur defect model.

Notes: The bone defect healing was assessed by micro-CT analysis and histological observation at 4 and 8 weeks postsurgery. (A) 3D reconstruction images were used to evaluate the new bone formation within femur defect in rats. Red bar $=200 \mu \mathrm{m}$. (B) Micro-CT parameters like BV/TV, Tb.N, Tb.Th, and Tb.Sp of the VOI were determined, respectively. (C) Histologic evaluation of $\mathrm{HE}$ stained paraffin-embedded tissue sections (amplification $200 \times$ ). The values are expressed as the mean \pm SD. $* P<0.05$ vs normal group.

Abbreviations: CUS, chronic unpredictable stress; CT, computed tomography; BV/TV, bone volume/trabecular volume ratio; Tb.N, trabecular number; Tb.Th, trabecular thickness; Tb.Sp, trabecular separation; VOI, volume of interest; HE, hematoxylin and eosin. 
the defect areas and the defects were completely filled with new bone in group $\mathrm{A}$. However, the volume of new bone was obviously less in group B (Figure 2C). These results indicated that rats with depression had a low ability of new bone formation, which suggested depression could attenuate bone healing.

\section{BMSCs derived from depressive rats had low osteogenic potential and autophagic level}

To further investigate the potential mechanism underlying the influence of depression on bone repair, the BMSCs were isolated from the rats with or without depression. Then, the P3 cells were cultured in an osteogenic differentiation medium to compare their osteogenic potential. After 21 days of osteogenic induction, the ALP staining intensity was obviously lower in depressive BMSCs, and normal BMSCs formed more mineralized matrix, as assessed by staining with ARS (Figure 3A). Corresponding to the staining observation, significantly higher expression levels of the osteogenic markers, COL I, OP, OC, and RUNX2, were detected in normal BMSCs by qRT-PCR (Figure 3B). These results suggested that BMSCs from depressive rats had lower osteogenic potential.

A previous study has indicated that autophagy plays an important role in osteoblasts, playing a role in mineralization

A
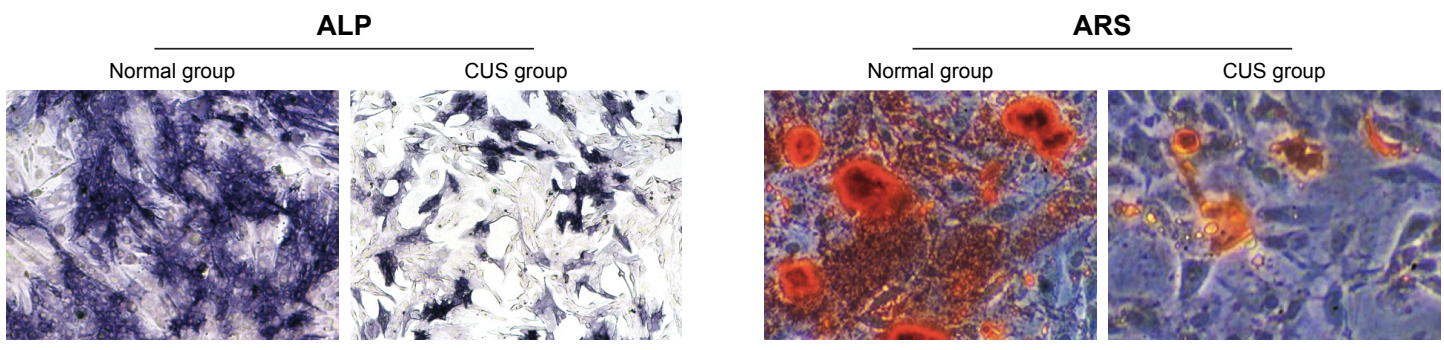

B

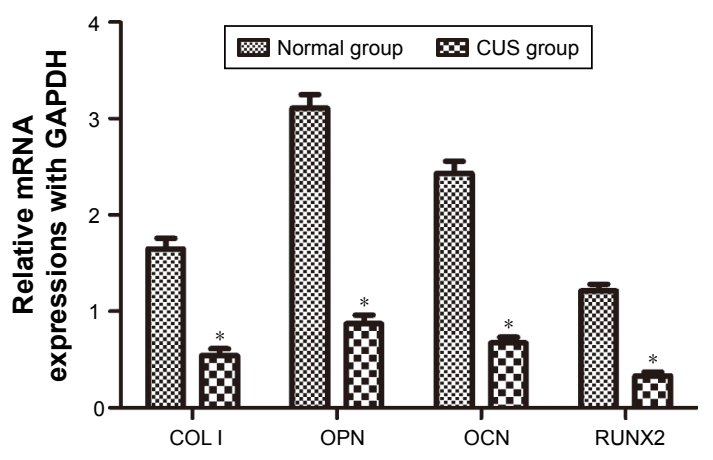

C

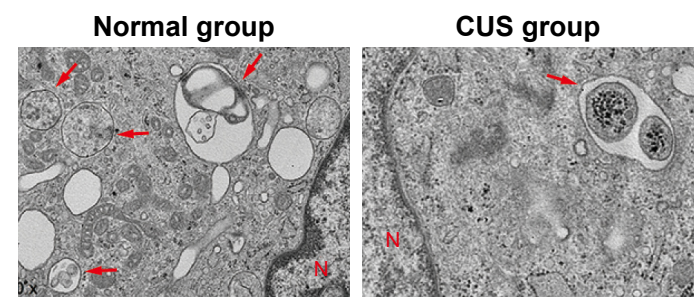

D
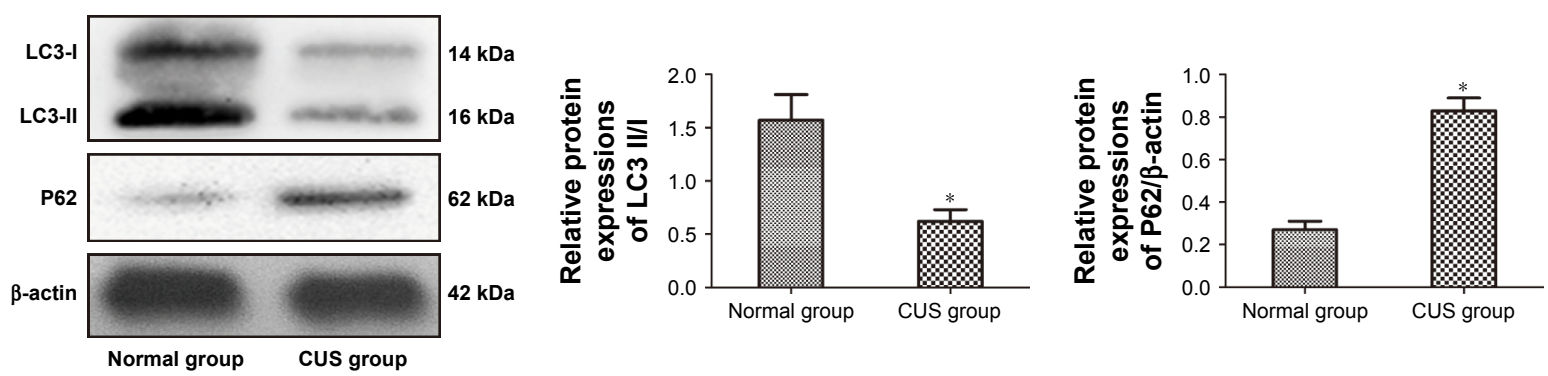

Figure 3 BMSCs derived from depressive rats had low osteogenic potential and autophagic level.

Notes: (A) Representative picture of ALP and ARS staining to detect osteogenic differentiation (amplification 200x). (B) The gene expressions of osteogenic markers COL I, OCN, OPN, and RUNX2 were examined by qPCR. Relative mRNA expressions were normalized to GAPDH. (C) Morphological observation of autophagy under TEM (amplification 15,000×). Red arrows represent the characteristic double-membranous ultrastructural morphology of autophagic vacuoles. $\mathrm{N}$ represents cell nucleus. (D) Western blot for the autophagy-related protein levels of LC3 and P62. The rate of LC3 II/I and P62/ $\beta$-actin represent the relative expressions. The values are expressed as the mean \pm SD. $* P<0.05$ vs normal group.

Abbreviations: ALP, alkaline phosphatase; ARS, Alizarin red S; CUS, chronic unpredictable stress; BMSCs, bone marrow mesenchymal stem cells; qPCR, quantitative polymerase chain reaction; TEM, transmission electron microscopy. 
and bone homeostasis. ${ }^{11}$ To assess the cellular basic autophagic level, the protein expressions of autophagic markers LC3 and P62 were detected prior to osteogenic induction. Western blot analysis showed that the depressive BMSCs significantly decreased the expression ratio of LC3II/I and increased the expression level of P62, which indicated that autophagy activity actually downregulated in BMSCs derived from depressive rats (Figure 3D). In addition, TEM observation demonstrated more typical double-membranous autophagosomes in normal BMSCs, which further proved there was a higher level of autophagy in normal BMSCs than in depressive BMSCs (Figure 3C).

\section{Autophagy activation promoted osteogenic differentiation of depressive BMSCs}

The abovementioned results suggested that low autophagic level was associated with the low osteogenic potential in depressive BMSCs. The autophagy agonist, RAP, was used to confirm that autophagy contributed to improvement of the osteogenic differentiation. As shown in Figure 4A, RAP significantly enhanced the autophagic level in depressive BMSCs, where the expression ratio of LC3II/I increased and the expression level of P62 decreased (Figure 4A). Furthermore, RAP stimulation enhanced osteoblast differentiation
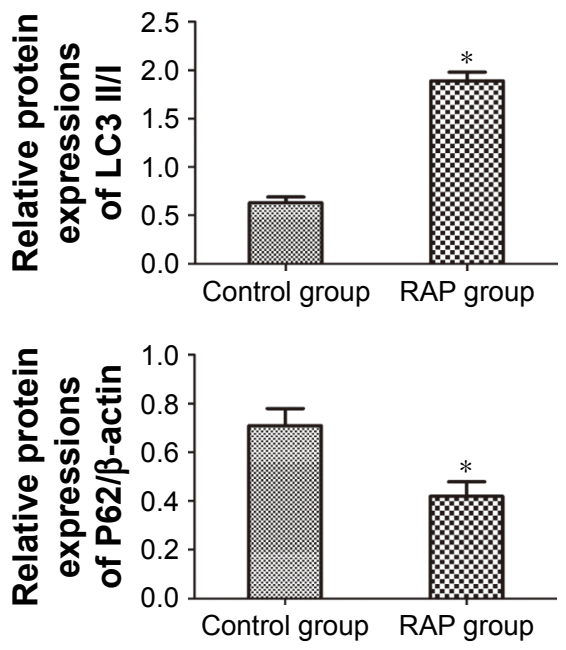

B

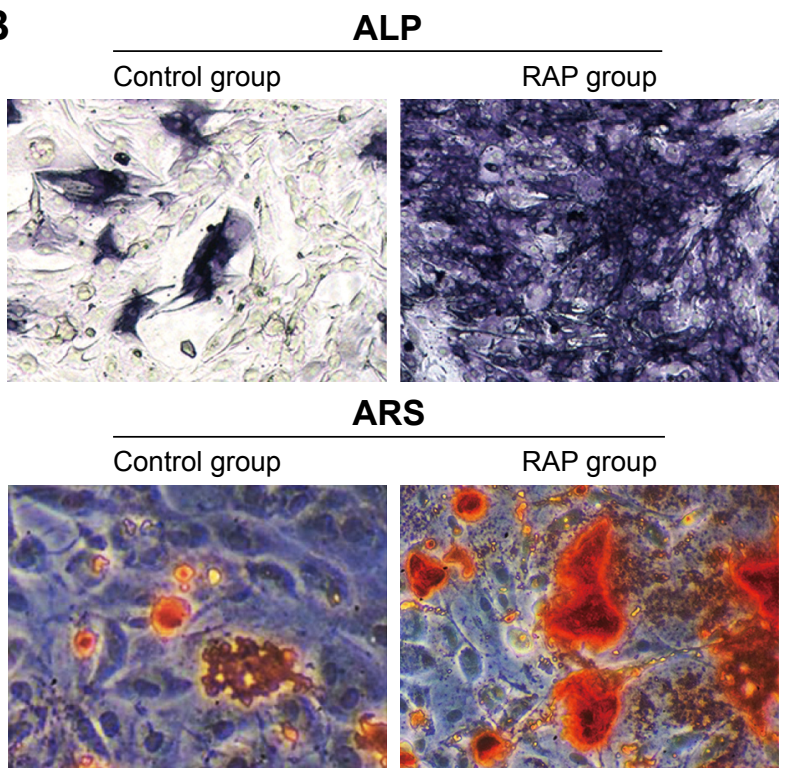

C

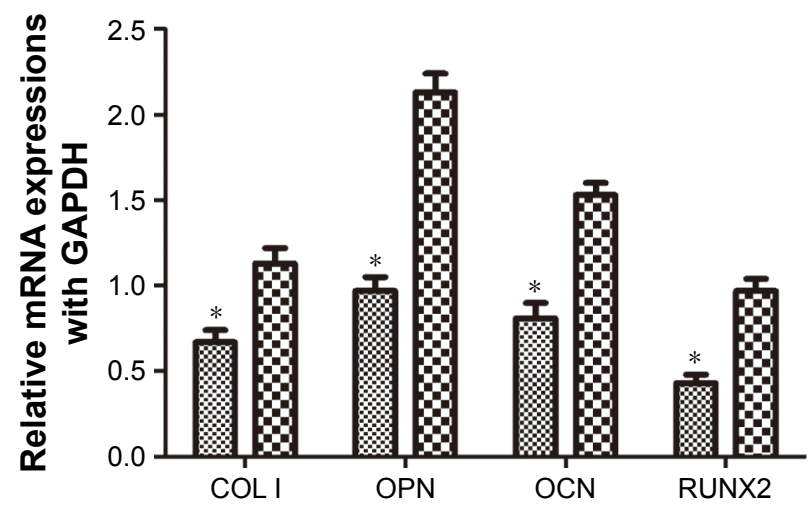

\$2 Control group RAP group

Figure 4 Autophagy activation promoted osteogenic differentiation of depressive BMSCs.

Notes: The depressive BMSCs were cultured in osteogenic differentiation medium in the presence of autophagy activator RAP. (A) Autophagic levels were detected by Western blot. (B) The levels of osteogenic differentiation were detected by ARS and ALP staining (amplification 200x). (C) The gene expressions of osteogenic marker COL I, OCN, OPN, and RUNX2 were examined by qPCR. Relative mRNA expressions were normalized to GAPDH. The values are expressed as the mean \pm SD. $* P<0.05$ vs control group.

Abbreviations: RAP, rapamycin; ALP, alkaline phosphatase; ARS, Alizarin red S; BMSCs, bone marrow mesenchymal stem cells; qPCR, quantitative polymerase chain reaction. 
in depressive BMSCs, as detected by ALP and ARS staining (Figure 4B), and increased osteogenic markers as detected by qRT-PCR (Figure 4C). These results indicated that osteogenesis differentiation could significantly improve through autophagy activation in BMSCs isolated from depressive rats.

\section{Discussion}

Previous studies have demonstrated that chronic stress and depressive disorder negatively affect bone mineral density and are closely related with high fracture incidence. ${ }^{3,12,13}$ The results of this study demonstrated for the first time that depressive disorder induced by CUS had an adverse effect on fracture healing through damage to the osteogenic differentiation of BMSCs in rats. Notably, low autophagic level was closely related with defect osteogenic ability in the BMSCs derived from depressive rats.

CUS has been widely used to establish depressive animal models. In the present study, SPT, FST, and OFT analyses confirmed that CUS resulted in a state of anhedonia and psychomotor retardation in rats, which were the major features of depressive behavior change. Depression is a common chronic affective disorder which has been robustly associated with physical function and mobility. ${ }^{14}$ Then, drill-hole injured rats were established to mimic the fracture condition. As for the sizes of bone defect, a previous study has proved that $<$ diameter $3 \mathrm{~mm}$ round bone defect is within the selfrepair capacity for rats. ${ }^{15}$ Micro-CT comparison between groups showed significant difference in fracture healing and callus formation. The normal rats showed accelerated callus formation rate in the drilled region of the cortical bone as early as 4 weeks. However, even at the end of 8 weeks postinjury, the depressive rats still failed to heal completely. The quantitative analysis using micro-CT showed that depression significantly attenuated the micro-CT parameters like BV/TV, Tb.N, and Tb.Th. The HE histological observation further confirmed that new woven bone was less often found in depressive group, which revealed that depressive rats had defects in osteogenesis.

Bone metabolism imbalance, especially osteoporosis, has been increasingly identified as an important comorbidity of depressive disorder. ${ }^{2}$ However, the underlying mechanism of their interaction remains unclear. Osteoporosis has the common pathological phenotype of defective BMSCs osteogenesis, ${ }^{16}$ since osteoblasts with osteogenetic potential were mainly differentiated from BMSCs. Thus, the osteogenic differentiation of BMSCs was further investigated within depressive and normal rats. Results showed that osteogenic potential of BMSCs from the depressive group was significantly impaired, as detected by ARS and ALP staining, of which ALP is expressed during the early stages of osteogenesis and ARS is expressed in more mature osteoblasts during matrix maturation and mineralization. ${ }^{17}$ The staining observation was corroborated by the transcript levels of osteogenic markers, like COL1A1, OPN, OCN, and RUNX2. The qPCR results showed that decreased expression of these osteogenic markers was determined in depressive BMSCs, which led to a consequence of poor fracture healing in depressive rats.

Autophagy is a genetically regulated and dynamic process associated with degradation and recycling of cellular components. ${ }^{18}$ The number of typical double-membranous autophagosomes under TEM observation is the golden standard to measure autophagic level. Furthermore, SQSTM1/ p62 has been shown to regulate autophagic degradation, and the conversion of cytosolic LC3-I to autophagic vesicle-associated LC3-II has been widely used to monitor autophagy flux. ${ }^{19}$ Various stresses could induce autophagy, the primary physiological function of which is to maintain cellular homeostasis. However, recent studies indicated that autophagy appeared to be related with differentiation of osteoblasts and osteoclasts. ${ }^{20}$ Our results also showed that autophagic level was significantly lower in depressive BMSCs, which was consistent with insufficient osteogenic capability. Wan et $\mathrm{al}^{5}$ clearly proved that autophagy activation could promote osteogenic differentiation of human BMSCs derived from osteoporotic vertebrae. To further detect the role of autophagy on osteogenesis differentiation, the pharmacological activator RAP was used to induce autophagy in depressive BMSCs. The results showed that RAP significantly promoted autophagy activation, which resulted in the upregulation of osteogenic differentiation. Thus, the present study provided evidence for a positive role of autophagy in osteoblast differentiation in BMSCs isolated from depressive rats.

The results of this study demonstrated low osteogenic potential and autophagic levels in BMSCs derived from the depressed rats, which led to attenuation of bone healing in the femur defect model. However, the present study has limitations related to lack of evidence to explain why the osteogenic potential and autophagic levels of BMSCs were low in the depression state. A previous study has demonstrated that the repeated stress hormone CORT in rats produced reliable behavioral and neurobiological alterations that paralleled many of the core symptoms and neurobiological changes associated with human depression. ${ }^{21}$ In addition, 
increasing evidence has proved that glucocorticoid signaling could significantly influence bone biology, ${ }^{22}$ of which autophagy as a target for glucocorticoid-induced osteoporosis is an important underlying mechanism. ${ }^{23}$ Therefore, corticosterone could be suggested as the key contact between depression and bone metabolism defect, and this deserves further study.

\section{Conclusion}

Our study found that depressive disorder was involved with poor fracture healing and also assessed the beneficial effects of autophagy on the cellular processes of osteogenic differentiation in depressive rats. Our findings provide valuable insight into the mechanism of bone metabolism disorder caused by depression and suggests that autophagy activation might be a novel treatment target for nonunion or delayed union of bone fracture in depressive patients.

\section{Disclosure}

The authors report no conflicts of interest in this work.

\section{References}

1. GBD 2015 DALYs and HALE Collaborators. Global, regional, and national disability-adjusted life-years (DALYs) for 315 diseases and injuries and healthy life expectancy (HALE), 1990-2015: a systematic analysis for the Global Burden of Disease Study 2015. Lancet. 2016; 388(10053):1603-1658.

2. Rosenblat JD, Gregory JM, Carvalho AF, McIntyre RS. Depression and disturbed bone metabolism: a narrative review of the epidemiological findings and postulated mechanisms. Curr Mol Med. 2016;16(2):165-178.

3. Bolton JM, Morin SN, Majumdar SR, et al. Association of mental disorders and related medication use with risk for major osteoporotic fractures. JAMA Psychiatry. 2017;74(6):641-648.

4. Garg P, Mazur MM, Buck AC, Wandtke ME, Liu J, Ebraheim NA. Prospective review of mesenchymal stem cells differentiation into osteoblasts. Orthop Surg. 2017;9(1):13-19.

5. Wan Y, Zhuo N, Li Y, Zhao W, Jiang D. Autophagy promotes osteogenic differentiation of human bone marrow mesenchymal stem cell derived from osteoporotic vertebrae. Biochem Biophys Res Commun. 2017;488(1):46-52.

6. Chen LM, Zhang AP, Wang FF, et al. Running exercise protects the capillaries in white matter in a rat model of depression. J Comp Neurol. 2016;524(17):3577-3586.
7. Brenes Saenz JC, Villagra OR, Fornaguera Trias J. Factor analysis of forced swimming test, sucrose preference test and open field test on enriched, social and isolated reared rats. Behav Brain Res. 2006; 169(1):57-65.

8. Tao ZS, Qiang Z, Tu KK, et al. Treatment study of distal femur for parathyroid hormone (1-34) and beta-tricalcium phosphate on bone formation in critical size defects in rats. J Biomater Appl. 2015;30(4): 484-491.

9. Yang JD, Cheng H, Wang JC, Feng XM, Li YN, Xiao HX. The isolation and cultivation of bone marrow stem cells and evaluation of differences for neural-like cells differentiation under the induction with neurotrophic factors. Cytotechnology. 2014;66(6):1007-1019.

10. Tang Z, Gong Z, Sun X. LncRNA DANCR involved osteolysis after total hip arthroplasty by regulating FOXO1 expression to inhibit osteoblast differentiation. J Biomed Sci. 2018;25(1):4.

11. Nollet M, Santucci-Darmanin S, Breuil V, et al. Autophagy in osteoblasts is involved in mineralization and bone homeostasis. Autophagy. 2014;10(11):1965-1977.

12. Calarge CA, Butcher BD, Burns TL, Coryell WH, Schlechte JA, Zemel BS. Major depressive disorder and bone mass in adolescents and young adults. J Bone Miner Res. 2014;29(10):2230-2237.

13. Hlis RD, McIntyre RS, Maalouf NM, Van Enkevort E, Brown ES. Association between bone mineral density and depressive symptoms in a population-based sample. J Clin Psychiatry. Epub 2017 Aug 22.

14. Yiend J, Paykel E, Merritt R, Lester K, Doll H, Burns T. Long term outcome of primary care depression. J Affect Disord. 2009;118(1-3):79-86.

15. Xie Z, Weng S, Li H, et al. Teriparatide promotes healing of critical size femur defect through accelerating angiogenesis and degradation of beta-TCP in OVX osteoporotic rat model. Biomed Pharmacother. 2017;96:960-967.

16. Stanovici J, Le Nail LR, Brennan MA, et al. Bone regeneration strategies with bone marrow stromal cells in orthopaedic surgery. Curr Res Transl Med. 2016;64(2):83-90.

17. Frith J, Genever P. Transcriptional control of mesenchymal stem cell differentiation. Transfus Med Hemother. 2008;35(3):216-227.

18. Mizushima N, Levine B, Cuervo AM, Klionsky DJ. Autophagy fights disease through cellular self-digestion. Nature. 2008;451(7182): 1069-1075.

19. Yoshii SR, Mizushima N. Monitoring and measuring autophagy. Int $J$ Mol Sci. 2017;18(9):pii: E1865.

20. Hocking LJ, Whitehouse C, Helfrich MH. Autophagy: a new player in skeletal maintenance? J Bone Miner Res. 2012;27(7):1439-1447.

21. Sterner EY, Kalynchuk LE. Behavioral and neurobiological consequences of prolonged glucocorticoid exposure in rats: relevance to depression. Prog Neuropsychopharmacol Biol Psychiatry. 2010;34(5): $777-790$.

22. Komori T. Glucocorticoid signaling and bone biology. Horm Metab Res. 2016;48(11):755-763.

23. Shen G, Ren H, Shang Q, et al. Autophagy as a target for glucocorticoidinduced osteoporosis therapy. Cell Mol Life Sci. Epub 2018 Feb 9.
Neuropsychiatric Disease and Treatment

\section{Publish your work in this journal}

Neuropsychiatric Disease and Treatment is an international, peerreviewed journal of clinical therapeutics and pharmacology focusing on concise rapid reporting of clinical or pre-clinical studies on a range of neuropsychiatric and neurological disorders. This journal is indexed on PubMed Central, the 'PsycINFO' database and CAS,

\section{Dovepress}

and is the official journal of The International Neuropsychiatric Association (INA). The manuscript management system is completely online and includes a very quick and fair peer-review system, which is all easy to use. Visit http://www.dovepress.com/testimonials.php to read real quotes from published authors. 